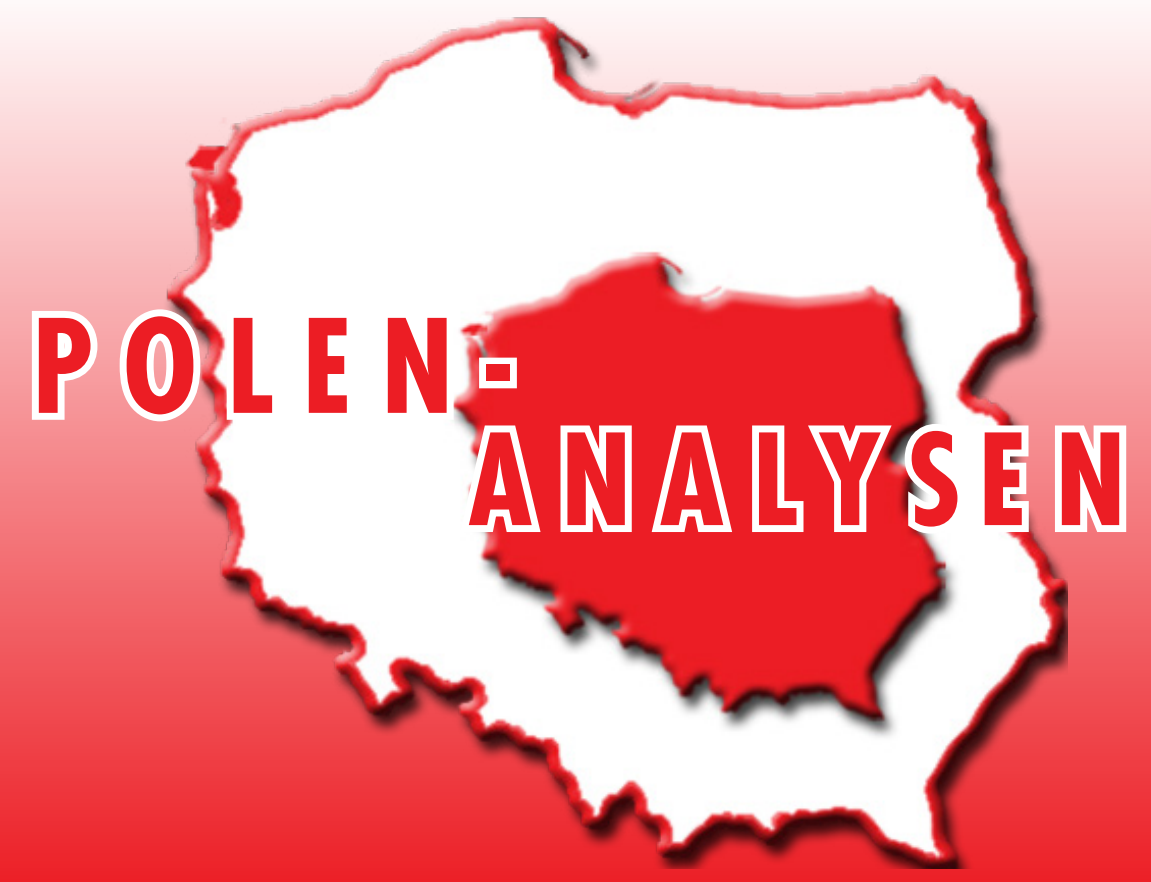

www.laender-analysen.de/polen

\title{
PARTEIENLANDSCHAFT IN BEWEGUNG
}

ANALYSE

Tauziehen auf der politischen Bühne - etablierte Parteien, neue Bündnisse und junge

Initiativen

Jarosław Flis, Krakau

- TABELLEN UND GRAFIKEN ZUM TEXT

Politische Einstellungen

CHRONIK

5. - 18. März 2019

\section{DEUTSCHES POLEN INSTITUT}

- Forschungsstelle Osteuropa an der Universität Bremen
Deutsche Gesellschaft

für Osteuropakunde e.V.
Zentrum für Osteuropa- und internationale Studien (ZOiS) gGmbH 


\section{Tauziehen auf der politischen Bühne - etablierte Parteien, neue Bündnisse und junge Initiativen}

Jarosław Flis, Krakau

\section{Zusammenfassung}

Die politische Bühne in Polen wird seit mehr als drei Jahren von der dominierenden Partei Recht und Gerechtigkeit (Prawo i Sprawiedliwość - PiS) und der Suche der Opposition nach einer erfolgversprechenden Strategie zur Rückgewinnung der politischen Initiative geprägt. Die Ergebnisse der Selbstverwaltungswahlen im Jahr 2018 bestätigten die größte Oppositionspartei, die Bürgerplattform (Platforma Obywatelska - PO), in der Strategie, für die Europawahlen im Mai 2019 die Europäische Koalition (Koalicja Europejska) zu bilden, ein breites Bündnis von der PO über Die Moderne (Nowoczesna) und die Polnische Bauernpartei (Polskie Stronnictwo Ludowe - PSL) bis zu der Demokratischen Linksallianz (Sojusz Lewicy Demokratycznej - SLD) u. a. Außerdem gründete der neue politische Star Robert Biedroń eine Partei jenseits des etablierten LinksRechts-Schemas unter dem Namen Frühling (Wiosna). Der sich abzeichnenden nächsten Phase politischer Auseinandersetzungen vor den Europawahlen im Mai und den Sejmwahlen im Herbst 2019 stellt der Autor den Wunsch vieler Polinnen und Polen nach einem gemäßigten Politikstil und nach Stabilität gegenüber.

$\mathrm{D}$ ie Jahre 2015 bis 2018 waren in der polnischen Politik eine Zeit gewaltiger Spannungen und gleichzeitig eine Phase, in der keine Wahlen auf Landesebene stattfanden, die ein Test des tatsächlichen Kräfteverhältnisses zwischen den politischen Gruppierungen hätten sein können. Auch wenn es viele für selbstverständlich hielten, war es keineswegs klar, dass die Machtkonstellation, die sich im Jahr 2015 gebildet hatte, Bestand haben würde. Damals hatte die Partei Recht und Gerechtigkeit (Prawo i Sprawiedliwość - PiS) die absolute Mehrheit im Sejm erlangt, teilweise dank glücklicher Zufälle. Diese ausnutzend, testete das Regierungslager unaufhörlich die Grenzen dessen, was in der öffentlichen Sphäre noch zulässig erschien und was nicht. Seine führenden Akteure lebten ganz offensichtlich in der Überzeugung, dass diese außergewöhnlichen Möglichkeiten die Gelegenheit seien, die erlangte Macht zu erweitern und sie zu verstetigen, gemäß dem Muster, das Viktor Orbán in Ungarn praktizierte. Dieses lüsterne Ausprobieren der regierenden Partei rief bei den Gegnern aufgebauschte Ängste hervor. Im Ergebnis beriefen sich beide Seiten in den Diskussionen über aktuelle Angelegenheiten in der Regel auf die schwerstwiegenden Argumente - die Fragen der Souveränität bzw. der Demokratie. Jede war von ihrer eigenen Überlegenheit und ihrer Fähigkeit überzeugt, die Waagschale vollkommen auf die eigene Seite zu neigen. Heute muss man dieses Bild der polarisierten Welt, in der jede kleine Schwingung für eine der Seiten den Fall in den Abgrund bedeuten könnte, als realitätsfern betrachten.

\section{Strategien für die Selbstverwaltungswahlen 2018}

Das Jahr 2018 brachte mit den Selbstverwaltungswahlen auf regionaler und lokaler Ebene den ersten und unanfechtbaren Test der realen Veränderungen im politischen Leben. Diese Wahlen riefen enorme Spannungen hervor. Man muss sich bewusst machen, dass die Selbstverwaltungswahlen in den Jahren 2010 und 2014 aufgrund einer ganzen Reihe von Umständen mit der eindeutigen Dominanz der damaligen Regierungskoalition aus Bürgerplattform (Platforma Obywatelska $P O$ ) und Polnischer Bauernpartei (Polskie Stronnictwo Ludowe - PSL) sowie der Marginalisierung der PiS verbunden gewesen waren. Darauf hatte eine Reihe von Faktoren Einfluss gehabt, die hier weiter auszuführen den Rahmen sprengen würde. Es bleibt die Tatsache, dass das aktuelle Regierungslager von dem Gefühl eines erlittenen Unrechts sowie von Zweifeln an der Wahlordnung der Selbstverwaltungswahlen begleitet wurde. Auf der anderen Seite hegte es große Hoffnungen, die Situation nun umzukehren. Der Sieg in den nationalen Wahlen im Jahr 2015 führte zur vollständigen Kontrolle aller Institutionen der Landespolitik, deren Höhepunkt die praktische Außerkraftsetzung des Verfassungsgerichts als unabhängiges Machtzentrum war. Das ließ auf Seiten des Regierungslagers die Hoffnung (und auf Seiten der Opposition die Angst) aufkeimen, dass es gelingen könnte, eine solche Situation auch im Bereich der Selbstverwaltung zu schaffen. Diese Hoffnungen und Ängste wurden zusätzlich durch zwei Faktoren verstärkt, durch Änderungen des Wahlrechts und durch das in dieser Form vorher nicht gekannte Engagement von Spitzenpolitikern im Wahlkampf auf der Selbstverwaltungsebene.

Die Wahlrechtsänderungen waren Ausdruck des bereits erwähnten Misstrauens der PiS-Mitglieder gegenüber den Regeln zur Durchführung der Wahlen. Neben einer Reihe eingeführter Änderungen, die potentielle Gefahren für die Ehrlichkeit der Wahlen bannen sollten, 
traten andererseits auch Vorschläge auf, die sich unschwer als Handlungen in die entgegengesetzte Richtung auffassen ließen, das heißt, die darauf ausgerichtet waren, die eigenen Wahlchancen zu vergrößern, indem das eigene Übergewicht ausgenutzt werden sollte. Ein Beispiel war die Idee, die Wahlkreise der Woiwodschaftslandtage (sejmiki) zu verkleinern, und das angesichts der Situation, dass die Opposition aufgesplittert war, während die Regierungspartei die größte Gruppierung war. Irgendwo zwischen diesen Lösungen waren die personelle Änderung und die neuen Berufungsregeln für den Vorsteher des Landeswahlbüros angesiedelt. Letztlich erfüllten sich weder die Hoffnungen noch die Ängste. Das Ausmaß der Änderungen des Wahlrechts wurde sehr stark begrenzt.

Ein ganz anderes Problem war das direkte Engagement der politischen Hauptakteure der nationalen Politik im Wahlkampf der Selbstverwaltungswahlen. Dies war vollkommen präzedenzlos und überbot sogar das Engagement Donald Tusks (PO) im Jahr 2010, als die $P O$ den Höhepunkt ihrer Erfolge erlebte. Es handelte sich aktuell nicht nur um Maßnahmen, die unverdächtig waren, wie die zahlreichen Auftritte des Ministerpräsidenten und anderer führender Politiker der stärksten Partei im ganzen Land. Zweifelhaft aus der Perspektive des demokratischen Ethos waren die dabei vertretenen Argumente und Andeutungen, dass es auf die Aktivitäten der Zentralregierung Einfluss haben werde, ob die Kandidaten der Regierungspartei in der betreffenden Stadt oder Region Erfolg haben würden. Dieser sollte eine Bedingung für das Wohlwollen von Seiten der Regierung beispielsweise im Falle von Investitionen sein. Aus dem unmittelbaren Engagement gingen auch sehr brutale Angriffe des Öffentlichen Fernsehens auf die PSL hervor, die bisher eine Schlüsselrolle in den Selbstverwaltungswahlen gespielt hatte.

Gleichzeitig führte das Bedrohungsniveau, das sich aus dem Umfragevorsprung und dem offenkundigen Appetit der regierenden Partei - und der seit drei Jahren andauernden Hysterie eines Teils des medialen Hintergrunds der Opposition - ergab, zu Änderungen in den Strategien der einzelnen Akteursgruppen. Die beiden ideologisch am engsten miteinander verbundenen Parteien, die PO und Die Moderne (Nowoczesna), bildeten die Bürgerkoalition (Koalicja Obywatelska - KO) und starteten gemeinsam in die Wahlen der sejmiki. Außerdem koordinierten sie weitreichend ihre Aktivitäten für die Direktwahlen der Ortsvorsteher, Bürgermeister und Stadtpräsidenten. Obgleich die PSL allein startete, wurde auch bei ihr die Koordination der Wahlkampfstrategien mit ihrem bisherigen Koalitionspartner in der Selbstverwaltung, der $P O$, sichtbar. Die Demokratische Linksallianz (Sojusz Lewicy Demokratycznej$S L D$ ) und die Bewegung Kukiz '15, die beiden übrigen
Parteien, die im Sejm oder auf regionaler Ebene präsent sind, starteten selbständig und verzichteten auf zusätzliche Aktivitäten, die auf die Erweiterung ihrer Wählerbasis zielten. Gleichzeitig trat die Initiative der Parteilosen Selbstverwalter (Porozumienie Bezpartyjnych Samorzadowców) in Erscheinung. Hier handelte es sich um einen Teil der lokalen Aktivisten, der sich von der politischen Spaltung im Land distanzierte, indem er sich für die Schaffung eines eigenen politischen Projektes engagierte und Wahllisten in allen 16 Woiwodschaften aufstellte.

\section{... und Ergebnisse}

Die Wahlergebnisse wurden mit einer sehr großen Anspannung erwartet. Die Ergebnisse der exit pollUmfragen und die nach und nach bekanntgegebenen Ergebnisse der Wahlkommissionen vermittelten einen sehr unklaren Eindruck. Aus der Distanz einiger Monate ist jedoch deutlich zu sehen, dass die Ergebnisse der Selbstverwaltungswahlen eine grundlegende Bedeutung für die Strategie der einzelnen Akteure hatten.

Die Selbstverwaltungswahlen, die zeitgleich auf der Ebene der Woiwodschaften, der Kreise und der Gemeinden stattfanden, liefern ein ungeheures Datendickicht. Die Daten erlauben, sehr leicht vollkommen entgegengesetzte Thesen aufzustellen. Was man allerdings aus ihnen extrahieren kann, ist die Nachricht, dass in diesen Wahlen das Gleichgewicht sowie der Status quo gewonnen haben. Das bedeutete auf der einen Seite die deutliche Verbesserung des Besitzstandes der PiS, insbesondere was die Woiwodschaftslandtage betrifft, wo sie bisher eine Randposition eingenommen hatte. Vollkommen erfolglos blieb allerdings die anfänglich formulierte und nur schrittweise während des Wahlkampfes korrigierte Absicht, die Opposition in den Landtagen an den Rand zu drängen. Das Ergebnis von 34 Prozent im Landesdurchschnitt bedeutete zwar einen optischen Sieg der PiS, doch lag dieser deutlich unter den Erwartungen sowie auch unter den Ergebnissen der landesweiten Umfragen. Dies ist auf die Spezifik der polnischen Selbstverwaltungswahlen als solche zurückzuführen sowie auf die Spezifik dieser konkreten Wahlen insbesondere. Das persönliche Engagement des Ministerpräsidenten Mateusz Morawiecki und des PiS-Parteichefs Jarosław Kaczyński führte vor allem zur Mobilisierung derjenigen Wähler, die gewöhnlich nicht an den Selbstverwaltungswahlen teilnehmen.

Diese zusätzliche Mobilisierung bedeutet zirka 1,5 Mio. zusätzliche Wähler im Vergleich zu den Wahlen im Jahr 2014, die nun ihre Stimmen abgaben; vor allem dort, wo die PiS bisher schwächere Ergebnisse erzielt hatte. Es lässt sich also vermuten, dass dies Wähler waren, die der PiS gegenüber negativ eingestellt waren. Das bedeutete keine große Wendung in der Unterstützung für die Opposition, die keinen großen Anlass hatte, 
ihr Ergebnis als Erfolg darzustellen. Die PiS aufgehalten zu haben, konnte sie allerdings als einen solchen behandeln, insbesondere im Lichte der Erfolge in den größten Städten inklusiv Warschau (Warszawa), die die Aufmerksamkeit der Medien auf sich zogen.

Die PiS erlangte die selbständige Mehrheit in sechs von 16 Woiwodschaften. In einer war sie in der Lage, eine Vereinbarung mit den Parteilosen Selbstverwaltern auszuhandeln, die die Rolle des Züngleins an der Waage spielten, in einer anderen, der Woiwodschaft Schlesien (województwo śląskie), erhielt sie die Mehrheit, weil sich ein Ratsmitglied aus den Reihen der Bürgerkoalition zurückzog. Einerseits rühmte sich die $P i S$ in ihren offiziellen Stellungnahmen des »eindeutigen« Sieges. Andererseits weisen jedoch die internen Äußerungen darauf hin, dass dieses Ergebnis wie ein Schwall kalten Wassers wirkte und bewusst machte, dass ein Machtverlust im Herbst 2019 sehr real ist.

Die PSL ging aus diesen Wahlen geschwächt hervor, aber die Überzeugung, dass sie von der negativen Kampagne der $P i S$ vernichtet werden würde, erwies sich als vollkommen übertrieben. Das Ergebnis von zwölf Prozent verband sich mit dem Machtverlust in den Hochburgen der PSL, wie den Woiwodschaften Lublin (woj. lubelskie) und Heiligkreuz (woj. świętokrzyskie), zeigte aber auch, dass die PSL deutlich lebendiger ist, als es in den letzten drei Jahren schien, als Kommentare auftauchten, die die Partei zum Abgang in die Vergessenheit verurteilten. Hier stellt sich die Frage, ob sich die Partei einem breiteren oppositionellen Block anschließen sollte.

Solche Zweifel tauchten bei der SLD nicht auf, die im Grunde so viele Stimmen wie bei den letzten Wahlen erhalten hat. Der höheren Wahlbeteiligung geschuldet, ergaben sich daraus allerdings ein deutlich geringerer Anteil an Unterstützung und deutlich weniger Mandate. Im Prinzip wurde die SLD eine Randgruppe in den sejmiki, auch wenn sie sich in zwei Woiwodschaften den dortigen Regierungskoalitionen anschließen konnte. Das Ergebnis auf dem Niveau von 6,5 Prozent war ein schwerer Schlag für alle, die auf den Wiederaufbau der Position der Linken nach der Niederlage bei den Parlamentswahlen im Jahr 2015 zählten bzw. darauf, dass sie die Rolle des Züngleins an der Waage übernehmen könnte.

Die Kukiz-Bewegung, also die Gruppierung, die am stärksten bemüht war, sich zwischen Regierungslager und radikaler Opposition zu positionieren, gab in den Wahlen ein sehr schwaches Bild ab. Zwar erhielt sie mit 5,62 Prozent im Landesdurchschnitt ein Ergebnis über der 5-Prozent-Hürde, bezogen auf die sejmiki bedeutete das allerdings, dass die Kukiz-Bewegung kein einziges Mandat erhielt. Die Unterstützung von etwas mehr als fünf Prozent bedeutete gleichzeitig, dass die Gruppie- rung jedes Jahr (seit den Sejmwahlen 2015) einen Prozentpunkt verloren hat. Die Beibehaltung dieses Trends heißt, dass sie sich bei den kommenden Wahlen unter der Fünfprozenthürde wiederfinden könnte. Dies ist ein deutlicher Hinweis auf das Problem dieser Gruppierung mit ihren Ideen und ihrer inneren Kohärenz was ein generelles Problem von Anti-System-Gruppierungen ist, die in das Parlament einziehen und damit den »Frische-Trumpf« verlieren, den sie in der Wahlkampfphase noch hatten.

Was das Verhältnis zwischen der $P O$ und Die Moderne betrifft, so ist hier eine interessante Bewegung auf Seiten der Opposition zu sehen. Die Aufrechterhaltung des Status quo in den Städten und nordwestlichen Woiwodschaften stärkte die Position des PO-Vorsitzenden Grzegorz Schetyna, führte aber gleichzeitig zu internen Spannungen im Projekt der Bürgerkoalition. Dies war verknüpft mit Spannungen innerhalb der Moderne, denn dort kam es zu einem internen Streit, ob man sich mit der $P O$ zusammentun oder ob man die eigene Identität bewahren solle. Dies führte praktisch dazu, dass die Partei auseinanderbrach und alsbald aufhörte, in den Umfragen eine Rolle zu spielen. Der Vorgang selbst bedeutete allerdings auch eine sehr ernste Störung der Glaubwürdigkeit der $P O$ als Organisatorin des Gegenlagers der PiS. Er erschwerte, die Gelegenheit beim Schopfe zu packen und eine breitere Verständigung aufzubauen, die von vielen Kommentatoren gefordert worden war und zumindest teilweise von der Arithmetik des polnischen Wahlsystems gerechtfertigt zu sein schien. Letztlich entstand die Europäische Koalition (Koalicja Europejska), der sich ebenfalls die PSL, die SLD wie auch Die Moderne anschlossen. Die gegenseitigen Beziehungen sind jedoch sehr vorsichtig, es fehlen Vertrauen und Enthusiasmus. Indessen zeigen die Umfragen allmählich sichtbare psychologische Gewinne dieser Initiative: Die PiS sah sich vor einem Gegner, der ihr mit Blick auf die gesellschaftliche Unterstützung, die er bündelt, ebenbürtig ist.

Als eine weitere Warnung für die PiS lassen sich die Ergebnisse der zweiten Wahlgänge der Bürgermeisterwahlen in den mittelgroßen Städten auffassen. Gerade diese Städte sind das beste Barometer für die politische Stimmung in Polen. Die Situation der PiS-Kandidaten in diesen Städten war im Jahr 2018 deutlich schlechter als vor vier oder acht Jahren. Die PiS verlor die Wahlen in einer Reihe von Städten, in denen sie vier Jahre zuvor noch gewonnen hatte. Offenbar ist dies das Ergebnis der Überschätzung der eigenen Kräfte und der Wahrnehmung der gesamten politischen Situation als einem "Match der PiS gegen den Rest der Welt«, wie es der Wahlkampfleiter der PiS, der Europaabgeordneten Tomasz Poręba, auf Twitter bezeichnete. Mehr als 
deutlich ist, dass die PiS entschieden zu schwach ist, um ein solches Match auf dem Selbstverwaltungssportplatz erfolgreich zu bestehen.

In den Wahlen auf Landesebene kann sich die Situation jedoch anders darstellen, da hier nicht der Faktor des Status quo in Gestalt der amtierenden Ortsvorsteher, Bürgermeister und Stadtpräsidenten ins Spiel kommt. Deutlich ist auch, dass die PiS ihre Unterstützung in den ländlichen Gebieten vergrößert hat. Es ist die Frage, inwieweit dies ein dauerhafter Erfolg ist, denn die Einwohner der ländlichen Gemeinden nehmen deutlich lieber an den Selbstverwaltungswahlen teil, als an denen auf Landesebene. Daher ist es keineswegs selbstverständlich, dass sie bei den Sejmwahlen im Herbst 2019 so zahlreich wählen gehen werden, wie 2018 bei den lokalen Wahlen. Die Wähler verstärkten aber ihre Beteiligung an den Wahlen, bei denen die landesweit vertretenen Parteien von Bedeutung sind, das heißt bei den Wahlen der sejmiki. Im Jahr 2018 verringerte sich die Anzahl der leeren Stimmzettel deutlich und ein wesentlicher Teil dieser Stimmen ging an die Wahllisten der PiS. Allerdings kann es sich hier auch um einen einmaligen Erfolg gehandelt haben.

\section{Affären im Regierungslager}

Die Selbstverwaltungswahlen allein wären ein ausreichender Schlag gewesen, notwendig für eine Veränderung in der polnischen Politik, aber sie waren bei weitem nicht der letzte Schlag. In den folgenden Monaten trat eine Reihe von Ereignissen ein und im Prinzip bringt jede Woche neue wichtige Erfahrungen mit sich. Die erste Welle bestand in einer Reihe von Affären, an denen Politiker des Regierungslagers beteiligt waren. Die Affäre bei der Finanzaufsicht (Komisja Nadzoru Finansowego) endete mit einem Rücktritt und später mit der Festnahme des Vorsitzenden dieser Institution wegen Korruptionsverdachts. Später kamen Kontroversen um die Vergütung der Assistentin des Präsidenten der Nationalbank (Narodowy Bank Polski) auf, und dieser zeigte sich vollkommen untalentiert, mit solchen Krisensituationen fertig zu werden.

Ein besonderer Fall ist die Kontroverse um die Investitionen in der Gesellschaft Srebrna, die mit der $P i S$ verbunden ist. Diese Angelegenheit ähnelt der "Abhöraffäre" während der Zeit der PO-PSL-Regierungskoalition. Die Informationen, die an die Öffentlichkeit gelangen, geben den Beteiligten das Gefühl, dass sie die Angelegenheit vernachlässigen und abwarten könnten, allerdings untergraben die immer neuen veröffentlichten Meldungen den Sinn dieser Strategie. Da sie aber einmal eingeschlagen wurde, ist es nun schwer, auf eine entschiedenere Art und Weise zu reagieren; umso mehr, weil manche Situationen vollkom- men eindeutig sind, wie die bei dieser Gelegenheit enthüllte Vergangenheit eines der engen Mitarbeiter von Jarosław Kaczyński und des Präsidenten des Nationalen Fonds für Umweltschutz (Narodowy Fundusz Ochrony Środowiska).

Die langfristigen Folgen der harten Verteidigungslinie gegen Beschuldigungen von Seiten der Opposition lassen sich auch am Beispiel einer anderen Affäre zeigen, die mit dem engen Mitarbeiter des ehemaligen Verteidigungsministers Antoni Macierewicz, Bartłomiej Misiewicz, zu tun hat, der aufgrund von Korruptionsvorwürfen inhaftiert wurde. Er war eine der ersten symbolischen Personen, die von der Opposition vor zwei Jahren angegriffen wurden. Anfangs wurde er vom gesamten Regierungslager entschieden verteidigt, später jedoch von seinem Amt abgezogen und schließlich inhaftiert, wahrscheinlich im Rahmen des Versuchs, medialen Schwierigkeiten für die Regierungspartei zuvorzukommen.

Seine Inhaftierung sollte sicherlich der öffentlichen Meinung signalisieren, dass "wir kein Mitleid mit den Unsrigen « haben. Im Lichte weiterer Ereignisse ist diese Botschaft jedoch weniger überzeugend als eine andere, die gleichzeitig auftrat. In der alternativen, direkt vom Justizministerium kontrollierten Interpretation agiert die Staatsanwaltschaft entsprechend den Interessen des Regierungslagers. Die allgemein von der Opposition formulierten Vorwürfe des Machtmissbrauchs werden nach alledem durch sehr konkrete Handlungen der Regierung bestätigt. Schon einmal, im Verlauf des Wahlkampfes im Jahr 2007, führte so etwas zu einer ungeheuren Mobilisierung und zur Machtenthebung der PiS. Es sieht so aus, als würde die regierende Partei immer noch denselben Versuchungen unterliegen und hätte keineswegs Schlüsse aus den damaligen Ereignissen gezogen.

\section{Das Projekt der Opposition: die Europäische Koalition}

Das, was ein gewisses Gleichgewicht auf der polnischen politischen Bühne herstellt, sind die fragwürdigen Strategien der Oppositionsparteien, die das Unvermögen des Regierungslagers begleiten. Der Aufstellung der Opposition gegenüber der regierenden Partei, wobei angenommen wird, dass deren Schwäche bereits ein ausreichender Grund sei, sie der Macht zu entheben, ohne gegen die eigenen Mängel vorzugehen, wird bei der Formierung der Europäischen Koalition sichtbar. Die Idee, das Engagement ehemaliger Ministerpräsidenten und Präsidenten zu einer der tragenden Säulen dieses Projekts zu machen, lässt sich schwerlich als gelungen beurteilen. Die Liste der Personen, die als Gesichter dieses Unternehmens vorgestellt werden, setzt sich aus Personen zusammen, die aus der Politik infolge eines Skan- 
dals oder verlorener Wahlen ausgeschieden sind. Dies ist eine deutliche Hinwendung zur Vergangenheit. Indessen zeigen die Erfahrungen mit den Selbstverwaltungswahlen in Städten wie Warschau oder Breslau (Wrocław), dass in Polen (wie an vielen anderen Orten in der Welt) der Grundsatz gilt, dass politische Parteien Vertrauen eher dadurch gewinnen, dass sie neue Kandidaten aufstellen, die frei von Lasten der Vergangenheit sind, als dadurch, dass sie sich auf Personen konzentrieren, die von der Vergangenheit gezeichnet sind.

\section{Die neue politische Gruppierung "Wiosna"}

Diese fragwürdigen Aktivitäten in Verbindung mit allgemein artikulierten Zweifeln am persönlichen Charisma Grzegorz Schetynas als $P O$-Parteichef ermöglichten die Entstehung einer neuen politischen Initiative, der Partei Frühling (Wiosna). Sie wird von Robert Biedroń, einem ehemaligen Abgeordneten der Palikot-Bewegung (Ruch Palikota) und anschließend Stadtpräsident von Stolp (Słupsk), geführt. Sie traf auf das Wohlwollen vieler liberaler Kommentatoren und wurde mit einem gut geplanten Timing vorher angekündigt. Zwar fallen die ersten Umfragen für die politische Initiative Frühling sehr günstig aus, doch gab es bereits ähnliche solche Fälle. Die polnische politische Bühne hat schon andere Initiativen hervorgebracht, die, als sie neu und frisch waren, Unterstützung in zweistelliger Prozenthöhe erreichten, aber später dahinschwanden und sich verschlissen, als die öffentliche Meinung detailliertere Erklärungen und Informationen erhielt.

Betrachtet man vergangene Wahlen in Polen, lässt sich feststellen, dass es eine Wählerschaft in bis zu zweistelliger Prozenthöhe gibt, die immer wieder bereit ist, neue Initiativen zu unterstützen, um ihren Widerspruch gegenüber dem Status quo und der aktuellen politischen Bühne zum Ausdruck zu bringen. Gleichzeitig sind die Wähler des linken Meinungsspektrums schon seit vielen Jahren von der Regierungsverantwortung ausgeschlossen und bleiben bei der Rivalität zwischen dem »liberaleren" Polen um die $P O$ herum und dem "solidarischen « Polen der PiS in der Opposition. Dies erklärt ihre deutliche Frustration und sehr starke emotionale Erregung, die in Untersuchungen politischer Einstellungen sichtbar werden, bei denen sich diese Gruppe durch ein hohes Maß an Geringschätzung gegenüber den politischen Gegnern auszeichnet.

Hier liegt also ein gewisses Potential. Die Verbindung beider Strömungen, das heißt der Protestwähler und der frustrierten Anhänger der Linken, könnte Chancen eröffnen. Allerdings wird hier ein deutliches Paradox sichtbar. Die Initiative Frühling wird als Versprechen eines völligen Umsturzes des aktuellen Kräfteverhältnisses und der Überwindung der Einteilung in
PO und PiS dargestellt. Dabei ist sie jedoch eindeutig gegen die PiS ausgerichtet, was die Überzeugung nährt, dass es weniger um die Überwindung dieser Spaltung geht, als vielmehr um die Ersetzung der einen Seite, mit dem Ziel, die andere zu Fall zu bringen. Diese ambitionierte Absicht erleichtert natürlich, mediale Ereignisse zu schaffen, sie weckt aber auch Erwartungen, die enttäuscht werden können, wenn die ersten, von der Frische der Initiative geprägten Umfragen von weiteren gefolgt werden, die eine stagnierende oder sogar geringer werdende Unterstützung zeigen.

Hinzu kommt: Die Präsentation der Initiative als etwas, das auf eine neue Qualität ausgerichtet ist, das heißt die Ausnutzung der Anti-Establishment-Emotionen, versperrt den Weg zu Verhandlungen mit den Vertretern der in der Politik bestehenden linken und liberalen Milieus. Angesichts der Gefahr, Wähler an die neue Initiative zu verlieren, zeigten sich diese geneigt, sich dem Lager der breiten Opposition unter dem Schild der $P O$ anzuschließen. Die $P O$ selbst müsste natürlich die Hauptquelle für die Abwanderung der Wähler zugunsten der neuen Initiative sein, damit diese tatsächlich die polnische Politik von Grund auf ändern könnte. Allerdings ist die $P O$ eine durch verschiedene Niederlagen abgehärtete Partei, die bereits seit längerer Zeit auf der politischen Bühne präsent ist. Die Beispiele Warschau und Breslau zeigen, dass sie enorme Möglichkeiten hat, neue Initiativen in Gang zu setzen und neue Personen hervorzubringen. Grzegorz Schetyna könnte Jarosław Kaczyńskis Manöver des Jahres 2015 wiederholen, das heißt in der vordersten Linie neue Personen aufstellen. Ihre stärkere Verwurzelung und das personelle Potential bedeuten, dass die $P O$ das Feld nicht kampflos räumen wird.

Die neue Initiative kann für diejenigen Wähler sehr attraktiv sein, die über die Aktivitäten des Regierungslagers, u. a. in weltanschaulicher Hinsicht, empört sind, doch ist darauf hinzuweisen, dass Wiosna sehr radikale Ansichten gegenüber dem aktuellen Status quo vertritt. Dabei geht es sowohl um weltanschauliche Fragen als auch um Vorhaben wie die Abrechnung mit der PiS. Der Vorschlag, eine Kommission einzurichten, deren Name an den der Kommission in der Republik Südafrika angelehnt ist, und Politikern der PiS mit dem Staatstribunal zu drohen, soll die Vermutung nähren, dass es sich um eine brutales und blutiges Regime handelt. Möglicherweise gibt eine solche Rhetorik die Chance, in der Politik zu existieren, auf jeden Fall aber erschwert sie die Umsetzung des ambitionierten Ziels, das sich die neue Initiative gesetzt hat.

Umfragen zeigen zudem, dass die Polen die Atmosphäre des Konfliktes leid sind, der von der PiS in den vergangenen drei Jahren warm gehalten wurde. Sie erwarten Ruhe und Stabilisierung, die die PiS nicht in der Lage zu garantieren war, denn sie war der Über- 
zeugung, dass ihr gerade der intensive Konflikt helfen würde. Es ist also schwer vorstellbar, dass die Wähler eine Gruppierung unterstützen, deren Programm und Erklärungen (auch wenn sie lächelnd vorgestellt werden) im Grunde die Ankündigung einer noch größeren Intensivierung der gesellschaftlichen Konflikte in Polen sind. Vielleicht wird diese Initiative daher die Rolle einnehmen, die heftigsten ideologischen Konflikte auf die Linie zwischen der $P i S$ und der Linken zu verlagern, was der $P O$ erlauben würde, die Wähler der Mitte und des Mitte-Rechts-Spektrums zu gewinnen, die sie bei den Sejmwahlen 2015 an die PiS verloren hatte. Das Problem ist hier nur, dass es unter den führenden und in den Medien präsenten $P O$-Politikern niemanden mehr gibt, der die gemäßigt konservative Mitte-Rechts-Strömung verkörpern könnte. Eine solche Persönlichkeit wäre hingegen der Chef der PSL, Władysław KosiniakKamysz, der in vielen Umfragen hohe Vertrauenswerte erhält, obgleich seine Partei nicht sehr stark ist.

\section{Entspricht der Politikstil den Wünschen der Wähler?}

Umfragen, die das Regierungslager in Auftrag gegeben hatte, zeigen ein weiteres Phänomen. Das, was in den sozialen Medien geschieht sowie in den überhitzten Medien, seien es öffentliche oder vom Regierungslager unterstützte private, entspricht nicht dem, was ein wesentlicher Teil der gemäßigten Wähler fühlt. Das aber sind diejenigen, die am meisten bereit sind, ihre Meinung angesichts bevorstehender Wahlen zu ändern. Vieles weist darauf hin, dass sie mit dem sozialpolitischen Programm der Regierung bereits zufrieden sind. Sie haben die Überzeugung gewonnen, dass sich die Regierung um die Allgemeinheit der Bürger kümmert und nicht nur um die, die am besten vorankommen. Daher wollen sie eher die Einstellung der Konflikte, die Reduzierung der Temperatur und eine ruhige, stabile Regierungstätigkeit. Das wiederum erinnert sehr an die ideologischen Annahmen der $P O$ am Anfang ihrer Regierungszeit unter Ministerpräsident Donald Tusk.

Sogar wenn es sich dieser Erwartungen bewusst ist, bleibt die Frage, ob das aktuelle Regierungslager in der Lage sein wird, ihnen gerecht zu werden. Hinderlich sind dabei die Erfahrungen seiner Aktivisten und deren Welt- sicht, die mit dem Kommunikationsstil von Jarosław Kaczyński übereinstimmen. Auch wenn seine Aussprüche seine heißesten Anhänger mobilisieren, so mobilisieren sie noch mehr seine Gegner. Sowohl Ministerpräsident Mateusz Morawiecki als auch Präsident Andrzej Duda scheinen nicht bereit zu sein, der PiS einen gemäßigteren Kommunikationsstil aufzuerlegen. Ihre Erklärungen in dieser Frage stehen immer wieder im Widerspruch zu darauf folgenden Äußerungen und Aktivitäten oder Initiativen von PiS-Politikern der zweiten Reihe oder des Parteichefs Kaczyński selbst.

All dies schafft ein sehr deutliches Gleichgewicht, für das die polnische Politik mit allen ihren Spannungen der vergangenen drei Jahre verantwortlich ist. Das Bewusstsein, dass die Kräfte der jeweiligen Lager sehr ausgeglichen sind, ist verbreitet. Im Prinzip schafft dieses Bewusstsein einen starken Impuls, sich zu vervollkommnen und konkrete Lösungen zu suchen - weniger damit verbunden, die andere Seite Ablehnung spüren zu lassen, sondern vielmehr damit, zu den gemäßigten Adressaten zu sprechen. Allerdings bewirken die zurückliegenden Jahre mit der ganz anders gearteten Atmosphäre, dass es den Politikern sehr schwer fällt, auf einen anderen Politikstil umzuschalten. Dies könnte die größte Gefahr für die Wirksamkeit sowohl des einen als auch des anderen Lagers sein. Es kann sich erweisen, dass die Fähigkeit, solche verhängnisvollen »Neigungen« zu vermeiden, darüber entscheiden wird, wer in den kommenden Wahlen erfolgreich sein wird.

Es scheint, als veranlasse die gesamte Situation zu einem gemäßigten Optimismus für die Zukunft. Nichts weist darauf hin, dass die Hoffnungen und Ängste, die sich in den letzten drei Jahren entfalteten, noch einmal in die polnische Politik zurückkehren werden. Es ist deutlich, dass sich die Wirklichkeit nicht so einfach ändert, wie es die fieberhaftesten Politiker gern hätten. Es erinnert dies an einen Wettkampf im Tauziehen. Das Tau selbst ist gespannt und beide Seiten zählen darauf, dass der nächste Ruck das Gleichgewicht ins Wanken bringt. Aber das Gleichgewicht bleibt trotz allem, und aus der Distanz betrachtet, ändert sich nicht so richtig viel.

Übersetzung aus dem Polnischen: Silke Plate

\section{Über den Autor}

Dr. habil. Jarosław Flis ist Soziologe am Institut für Verwaltung und gesellschaftliche Kommunikation an der Jagiellonen-Universität in Krakau (Uniwersytet Jagielloński, Kraków). Seine Forschungsschwerpunkte sind Soziologie der Politik, Public Relations, Wahlen, ihre Regularien und ihr Einfluss auf die politischen Akteure. Er war Mitglied der Expertenkommission zur Wahlordnung, die im Jahr 2011 beschlossen wurde. Er verfasst regelmäßig Beiträge und Kommentare für Fernsehen und Printmedien. 


\section{Politische Einstellungen}

Grafik 1: Die politischen Einstellungen der Polinnen und Polen (Februar 2019, \%)

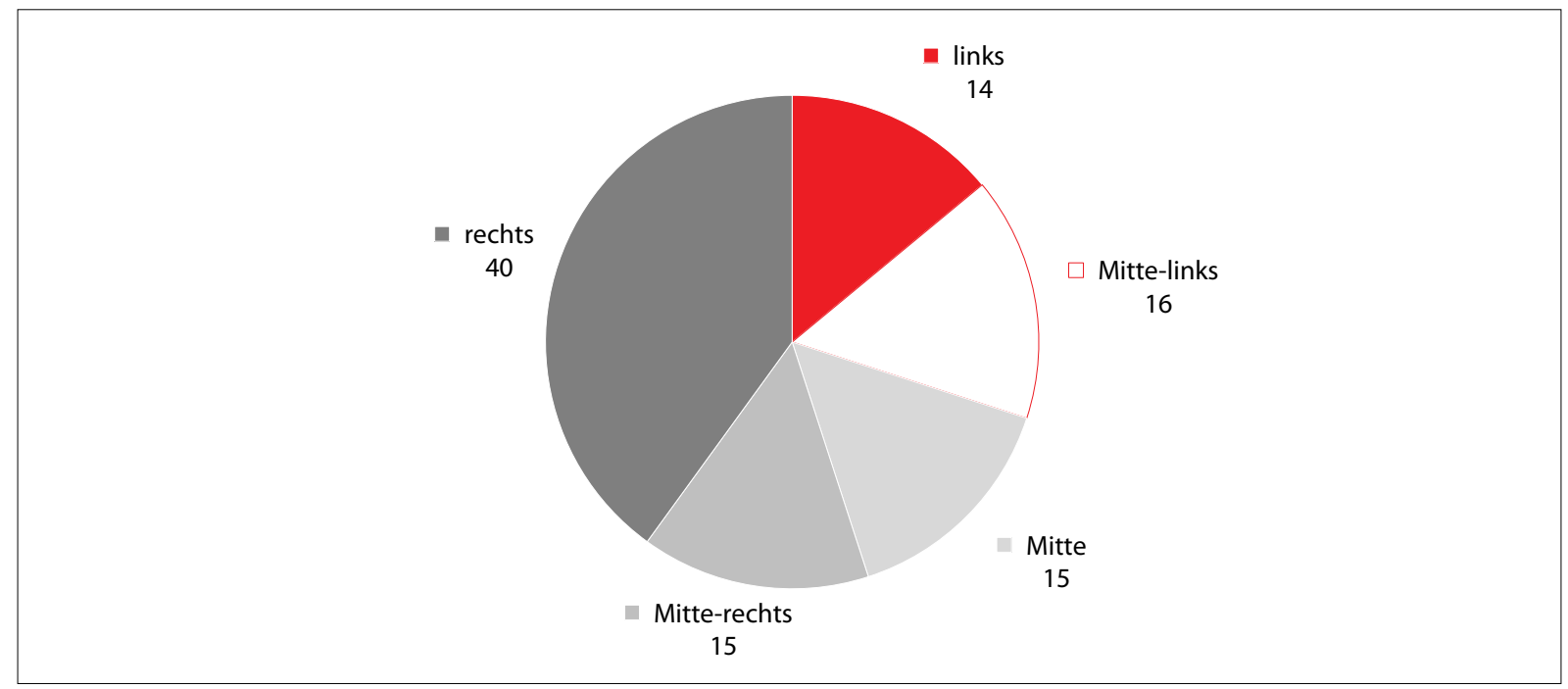

Quelle: Repräsentative Umfrage von IPSOS für OKO.press vom 14. bis 16.02.2019. https://oko.press/balast-prawicy-polsku-przechyla-polityke-ale-ta-sila-pisjest-tez-slaboscia-sondaz-oko-press/ (abgerufen am 18.03.2019).

\section{Grafik 2: Die Unterstützung für die Parteien (Februar 2019, \%)}

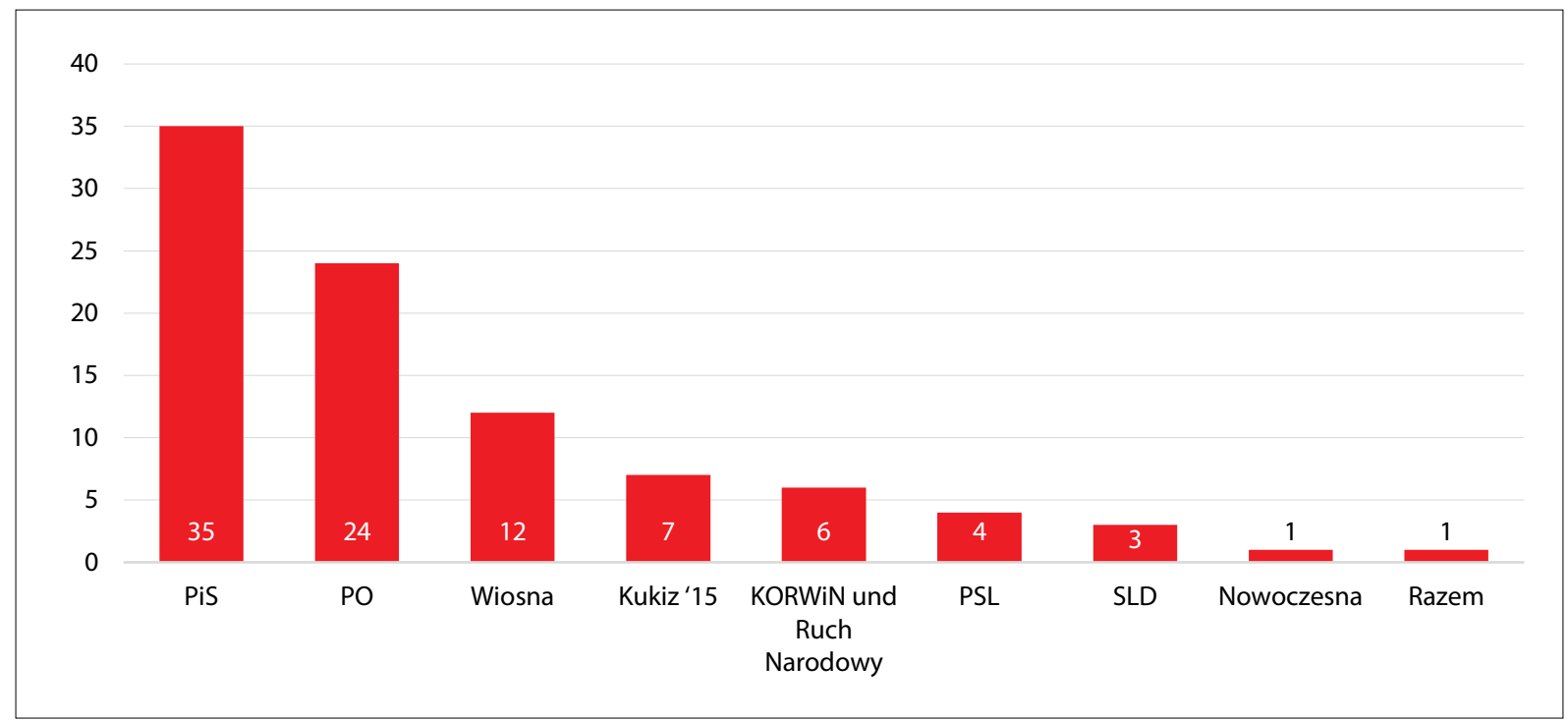

PiS/Prawo i Sprawiedliwość - Recht und Gerechtigkeit; PO/Platforma Obywatelska-Bürgerplattform; Wiosna - Frühling; KORWiN/Koalicja Odnowy Rzeczypospolitej Wolność i Nadzieja - Koalition der Erneuerung der Republik Freiheit und Hoffnung; Ruch Narodowy - Nationale Bewegung; PSL/Polskie Stronnictwo Ludowe Polnische Bauernpartei; Sojusz Lewicy Demokratycznej - Demokratische Linksallianz; Nowoczesna - Die Moderne; Razem - Gemeinsam

Quelle:Repräsentative Umfrage von IPSOS fürOKO.press vom 14. bis 16.02.2019. https://oko.press/dlaczego-pis-owi-nie-spada-otoz-spada-i-to-mocno-a-komuzabiera-biedron-sondaz-oko-press/ (abgerufen am 18.03.2019). 


\section{Grafik 3: Die Wählerwanderung zur Partei »Wiosna« (Februar 2019, in Prozentpunkten)}

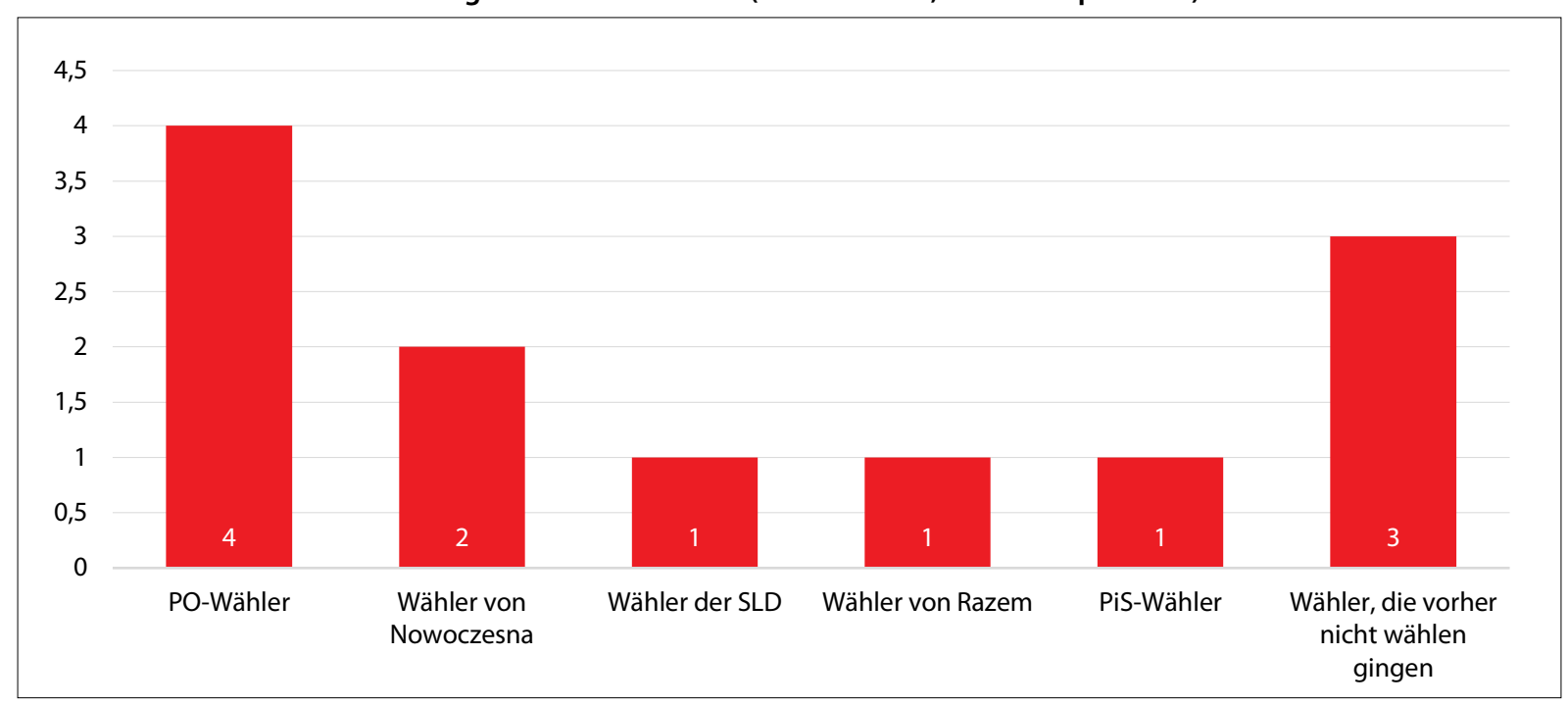

PO/Platforma Obywatelska - Bürgerplattform; Nowoczesna - Die Moderne; Sojusz Lewicy Demokratycznej - Demokratische Linksallianz; Razem - Gemeinsam; PiS/Prawo i Sprawiedliwość - Recht und Gerechtigkeit

Quelle: Repräsentative Umfrage von IPSOS fürOKO.press vom 14. bis 16.02.2019. https://oko.press/dlaczego-pis-owi-nie-spada-otoz-spada-i-to-mocno-a-komuzabiera-biedron-sondaz-oko-press/ (abgerufen am 18.03.2019).

Grafik 4: Die Unterstützung für die fünf wichtigsten politischen Kräfte entsprechend den politischen Einstellungen der Befragten (Februar 2019, \%)

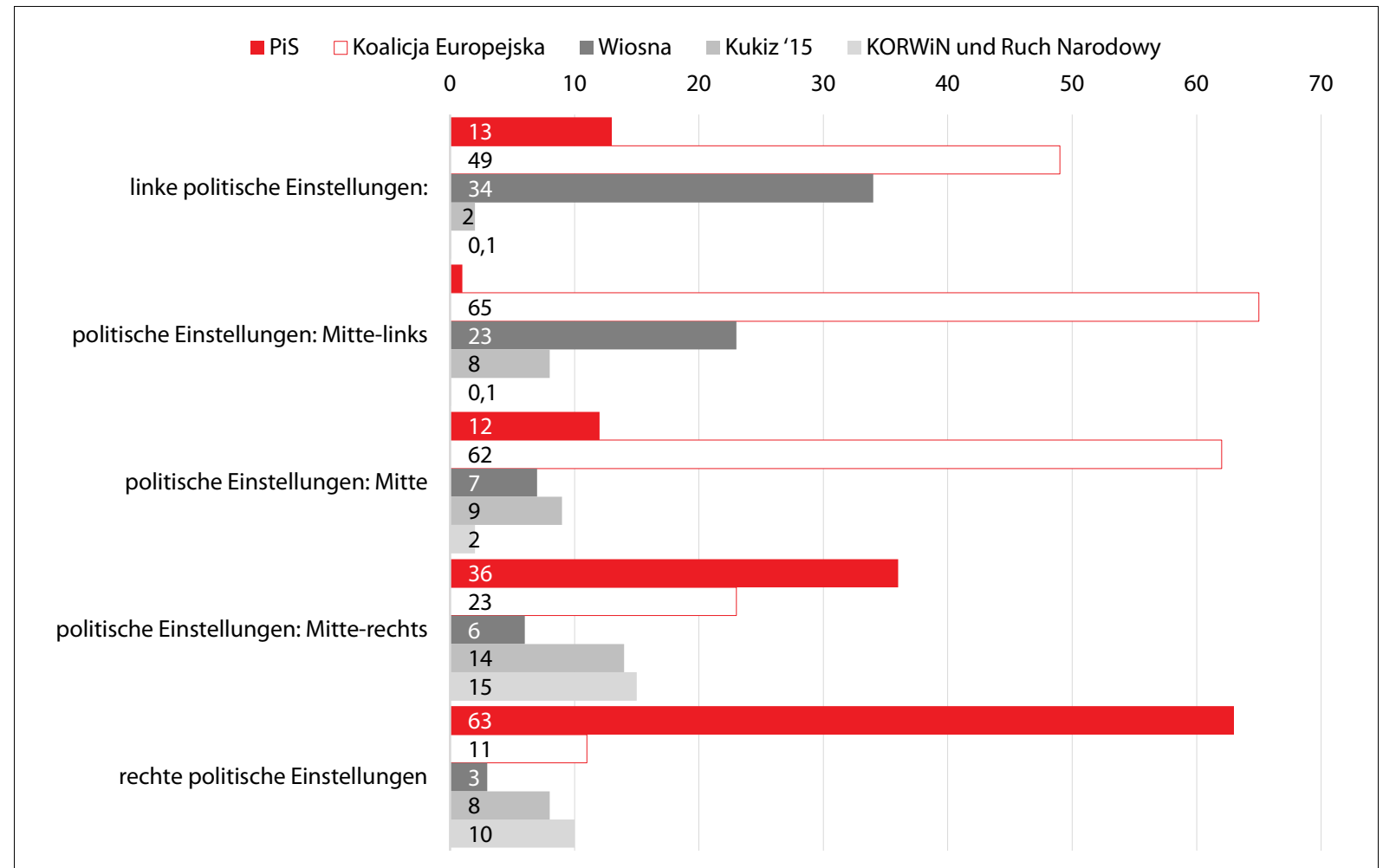

PiS/Prawo i Sprawiedliwość - Recht und Gerechtigkeit; Koalicja Europejska - Europäische Koalition (Zusammenschluss aus: PO/Platforma Obywatelska - Bürgerplattform; Sojusz Lewicy Demokratycznej-Demokratische Linksallianz; Nowoczesna - Die Moderne; PSL/Polskie Stronnictwo Ludowe - Polnische Bauernpartei; Zieloni-Die Grünen; Teraz - Jetzt); Wiosna - Frühling; KORWiN/Koalicja Odnowy Rzeczypospolitej Wolnośći Nadzieja-Koalition der Erneuerung der Republik Freiheit und Hoffnung; Ruch Narodowy - Nationale Bewegung;

Quelle: Repräsentative Umfrage von IPSOS für OKO.press vom 14. bis 16.02.2019. https://oko.press/balast-prawicy-polsku-przechyla-polityke-ale-ta-sila-pisjest-tez-slaboscia-sondaz-oko-press/ (abgerufen am 18.03.2019). 
Grafik 5a: Wer will die Partei »Wiosna« wählen? (nach Geschlecht; \%)

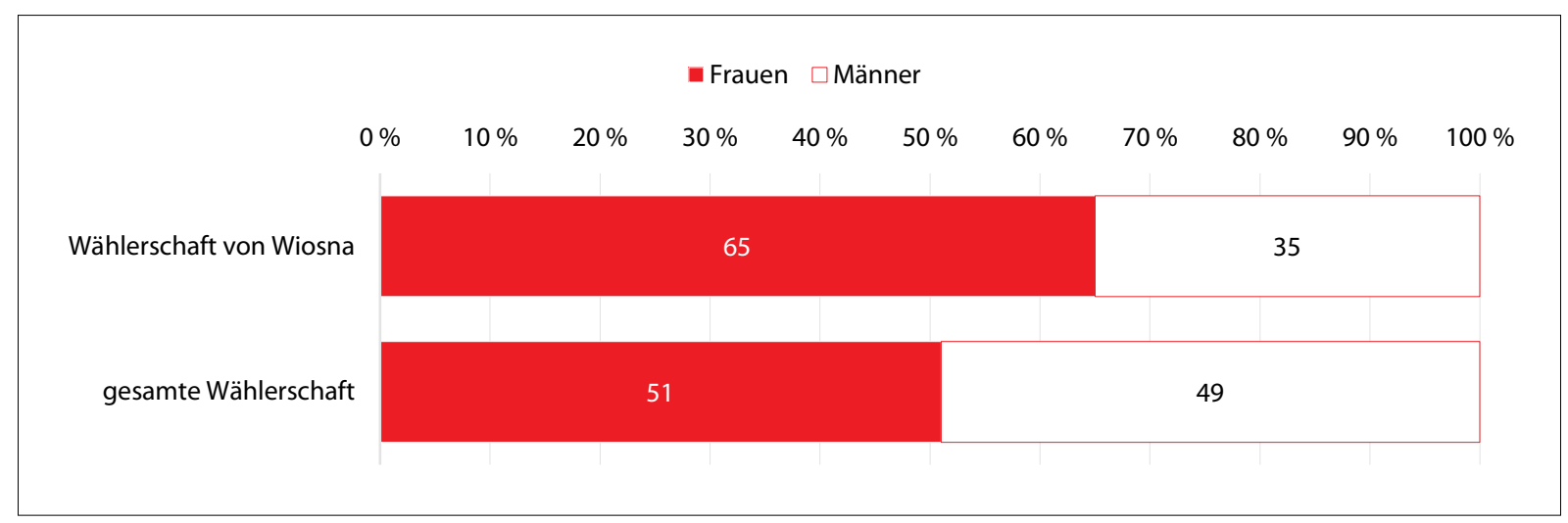

Quelle: CBOS: Komunikat z Badań Nr. 26/2019: Kto chce głosować na Wiosnę? [Wer will die Partei »Frühling« wählen?]. 02/2019. www.cbos.pl

Grafik 5b: Wer will die Partei »Wiosna« wählen? (nach Altersgruppen; \%)

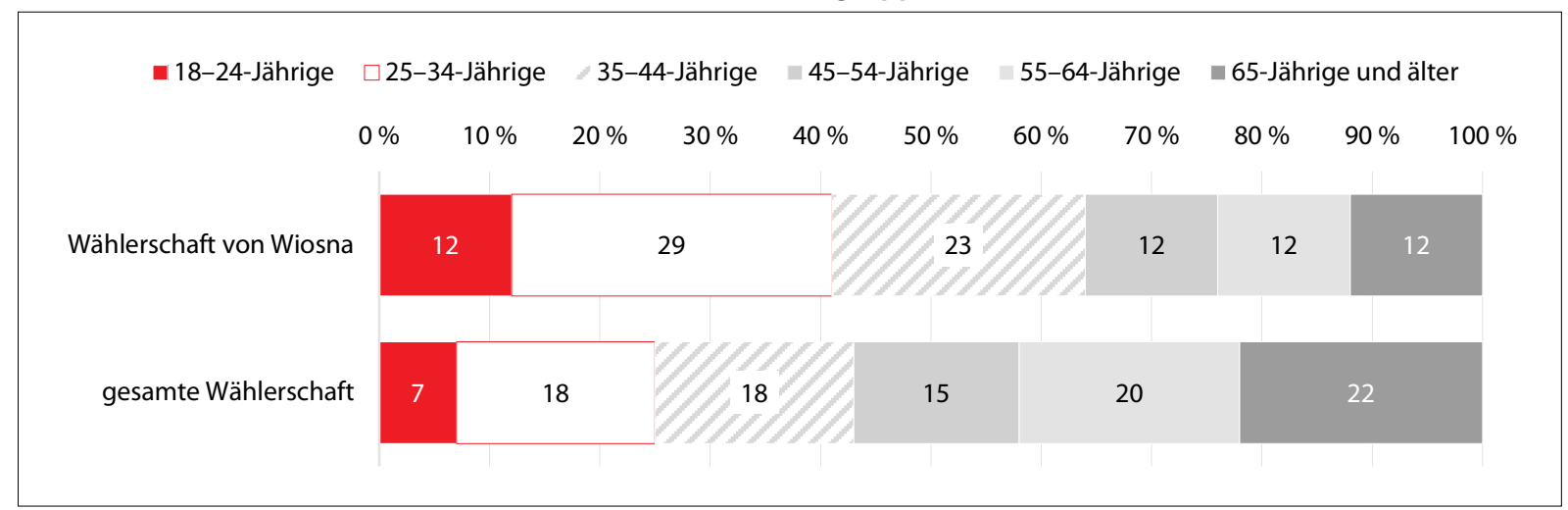

Quelle: CBOS: Komunikat z Badań Nr. 26 /2019: Kto chce głosowaćna Wiosnę? [Wer will die Partei »Frühling« wählen?]. 02/2019. www.cbos.pl

Grafik 5c: Wer will die Partei »Wiosna« wählen? (nach Größe des Wohnorts; \%)

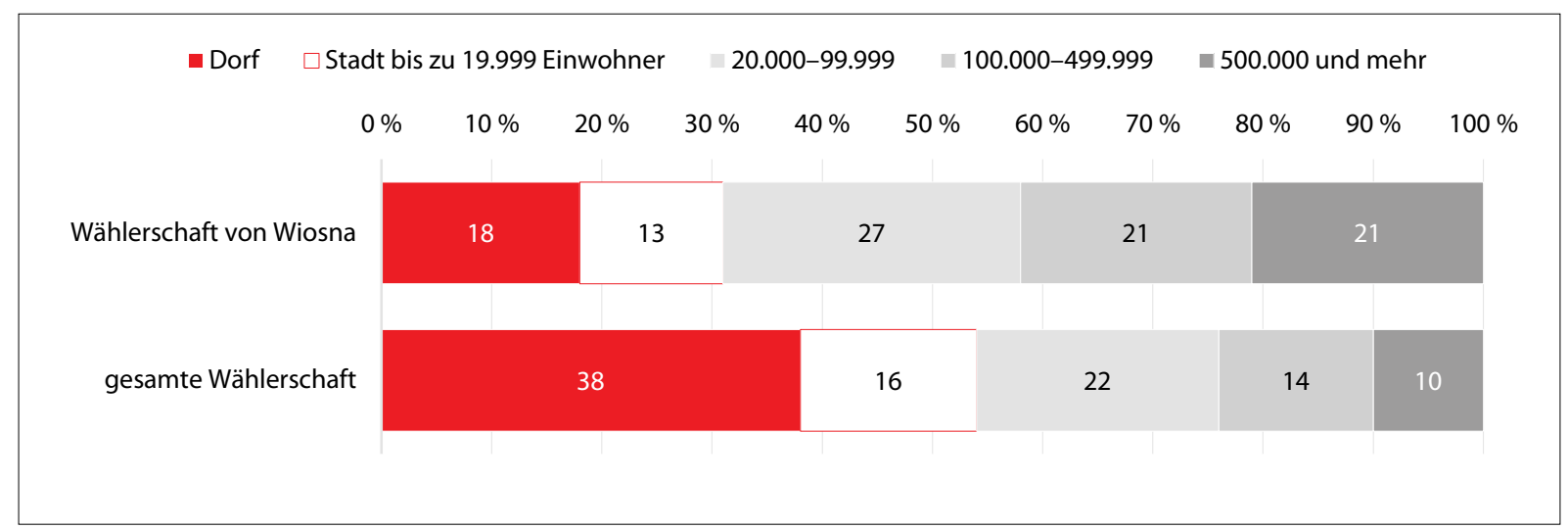

Quelle: CBOS: Komunikat z Badań Nr. 26/2019: Kto chce głosować na Wiosnę? [Wer will die Partei »Frühling« wählen?]. 02/2019. www.cbos.pl 


\section{5. - 18. März 2019}

\begin{tabular}{|c|c|}
\hline 05.03.2019 & $\begin{array}{l}\text { Ministerpräsident Mateusz Morawiecki teilt mit, dass dem Sejm in den kommenden zwei Monaten Gesetzes- } \\
\text { entwürfe zur Auszahlung einer zusätzlichen Rentenzahlung an alle Bezieher und zum Wiederausbau des Perso- } \\
\text { nennahverkehrs auf Gemeinde- und Kreisebene vorgelegt würden. Beide Vorhaben gehören zu den fünf neuen } \\
\text { sozialpolitischen Programmen, die Recht und Gerechtigkeit (Prawo i Sprawiedliwość- PiS) auf ihrem Parteitag } \\
\text { im Februar angekündigt hatte, und sollen aus den Einnahmen aus der Bekämpfung des Betrugs bei der Mehr- } \\
\text { wertsteuer finanziert werden. Kritiker sprechen von Wahlgeschenken an Rentner vor den Europawahlen im Mai. }\end{array}$ \\
\hline 06.03 .2019 & $\begin{array}{l}\text { Elżbieta Rafalska, Ministerin für Arbeit und Sozialpolitik, gibt die aktuellen Arbeitslosenzahlen bekannt. Im } \\
\text { Februar } 2019 \text { waren 1,018 Mio. Personen arbeitslos. Die Arbeitslosenquote betrug ebenso wie im Januar 6,1\%. } \\
\text { Im Vergleich zum Februar } 2018 \text { sank die Quote um 0,7 Prozentpunkte. }\end{array}$ \\
\hline 07.03.2019 & $\begin{array}{l}\text { Nach Angaben von Eurostat wurden im Jahr } 2017 \text { in Polen } 47 \% \text { der Leitungspositionen auf mittlerer Ebene } \\
\text { von Frauen besetzt (EU: } 36 \% \text {; Deutschland: } 30 \% \text { ). In Polen betrug die Differenz zwischen den Beschäfti- } \\
\text { gungsraten von Männern und Frauen im Jahr } 2017 \text { zugunsten der Männer 14,6 Prozentpunkte (EU: 11,5\%; } \\
\text { Deutschland: 7,9\%). }\end{array}$ \\
\hline 08.03.2019 & $\begin{array}{l}\text { Aus Anlass des 20. Jahrestages des Beitritts Polens zur NATO und des 70jährigen Bestehens des Verteidigungs- } \\
\text { bündnisses findet in Warschau eine Konferenz statt. Teilnehmer einer Podiumsdiskussion sind Außenminister } \\
\text { Jacek Czaputowicz, NATO-Generalsekretär Jens Stoltenberg und die ehemalige US-Verteidigungsministerin } \\
\text { Madeleine Albright. Czaputowicz mahnt bessere Maßnahmen und Reaktionen auf hybride Gefahren wie Ter- } \\
\text { rorismus und Desinformation und im Bereich der Cybersicherheit an. }\end{array}$ \\
\hline 10.03 & $\begin{array}{l}\text { Die Ministerpräsidenten und Verteidigungsminister der Visegrád-Staaten (Polen, Tschechien, Slowakei, Ungarn) } \\
\text { besuchen in der "Woche der NATO« zum 20jährigen Beitritt der Staaten zur NATO (12. März) die 1. Panzer- } \\
\text { brigade in Warschau. Am Appell nehmen auch Soldaten der in Polen stationierten Partnerländer teil, und es } \\
\text { findet eine Schau militärischer Fahr- und Flugzeuge statt. Ministerpräsident Mateusz Morawiecki hebt hervor, } \\
\text { dass es die einmalige Eigenschaft der NATO sei, eine gemeinsame Verteidigung zu schaffen, ohne in die sou- } \\
\text { veränen Rechte des jeweiligen Mitgliedslandes einzudringen. }\end{array}$ \\
\hline 12.0 & $\begin{array}{l}\text { Bildungsministerin Anna Zalewska trifft sich in Warschau mit Vertretern der Gewerkschaft Solidarność. In } \\
\text { Krakau besetzen in der Solidarność organisierte Lehrer seit zwei Tagen die Schulaufsichtsbehörde. Die Gewerk- } \\
\text { schaft fordert u. a. höhere Lehrergehälter, die Gewerkschaft der Polnischen Lehrerschaft (Związek Nauczyciel- } \\
\text { stwa Polskiego - ZNP) schließt einen Generalstreik der Lehrer im April nicht aus. }\end{array}$ \\
\hline 13.03 & $\begin{array}{l}\text { In Warschau blockieren am Morgen einige Dutzend Landwirte den Verkehr auf dem Plac Zawiszy und zün- } \\
\text { den Autoreifen an. Es kommt zu Festnahmen durch die Polizei. Nach Angaben des Pressebüros der Warschauer } \\
\text { Stadtverwaltung war der Protest nicht angekündigt worden. Zu dem Protest hatte die landwirtschaftliche Inte- } \\
\text { ressenvertretung AGROunia aufgerufen; bereits seit November } 2018 \text { hatten in verschiedenen Städten Polens } \\
\text { Protestaktionen stattgefunden. Michał Kołodziejczak, Mitgründer der AGROunia, sagt, die Proteste richteten } \\
\text { sich dagegen, dass die polnischen Landwirte vom nationalen und internationalen Markt abgeschnitten wür- } \\
\text { den. Gefordert würde die Kennzeichnung von Lebensmitteln, die in Polen hergestellt würden, in großflächi- } \\
\text { gen ausländischen Ladenketten sollen mindesten } 51 \% \text { der Produkte aus Polen kommen. Weiter fordert er, dass } \\
\text { landwirtschaftliche Gewerkschaften, Firmen und Organisationen ihre Gelder transparent abrechnen müssten, } \\
\text { sowie Unterstützung für die Obst- und Gemüsebauern und die Schweinemäster. }\end{array}$ \\
\hline 14.03.2019 & $\begin{array}{l}\text { In seinem Exposé zur Außenpolitik vor dem Sejm weist Außenminister Jacek Czaputowicz entschieden den } \\
\text { Vorschlag der Europäischen Kommission zurück, die Auszahlung von EU-Mitteln an einen Mitgliedsstaat von } \\
\text { der Bewertung des Zustands seiner Rechtsstaatlichkeit abhängig zu machen. Die EU-Mitgliedsländer hätten } \\
\text { der Europäischen Kommission und dem Europäischen Rat nur die Kompetenz verliehen, solche Angelegenhei- } \\
\text { ten vor den Europäischen Gerichtshof zu bringen, und hätten nicht das Recht, selbst zu richten. Die Auszah- } \\
\text { lung der EU-Mittel sei durch den EU-Vertrag verpflichtend. }\end{array}$ \\
\hline 14.03 .2019 & $\begin{array}{l}\text { Der Polnische Episkopat stellt Daten zum sexuellen Missbrauch von Minderjährigen in der katholischen Kir- } \\
\text { che in Polen vor. Demnach haben zwischen dem 1. Januar } 1990 \text { und dem 30. Juni } 2018382 \text { Geistliche Min- } \\
\text { derjährige sexuell missbraucht, insgesamt sollen } 625 \text { Opfer betroffen sein. }\end{array}$ \\
\hline 15.03 .2019 & $\begin{array}{l}\text { Der Sejm verabschiedet mit } 376 \text { Stimmen (zwei Gegenstimmen, zwei Enthaltungen) ein Gesetz zur Anerken- } \\
\text { nung von in Großbritannien erworbenen beruflichen Qualifikationen im Falle eines harten Brexit. Bereits aner- } \\
\text { kannte Qualifikationen werden in Polen weiter Gültigkeit haben. }\end{array}$ \\
\hline
\end{tabular}




\begin{tabular}{|l|l|}
\hline 17.03.2019 & $\begin{array}{l}\text { Der Vorsitzende der Gewerkschaft der Polnischen Lehrerschaft (Związek Nauczycielstwa Polskiego - ZNP), } \\
\text { Sławomir Broniarz, unterstreicht in einer schriftlichen Erklärung die Entschlossenheit der Lehrer zum Streik, } \\
\text { da die Regierung nicht auf ihre Forderungen eingehe, sondern in Aussicht stelle, die protestierenden Lehrer zu } \\
\text { ersetzen. Dem Bildungssystem drohe der Kollaps infolge von Unterfinanzierung. Die niedrigen Gehälter wür- } \\
\text { den die Lehrer veranlassen, sich andere Arbeitsstellen zu suchen. Zurzeit führt die ZNP die Abstimmung über } \\
\text { einen Streik unter ihren Mitgliedern durch. }\end{array}$ \\
\hline 18.03.2019 & $\begin{array}{l}\text { Ministerpräsident Mateusz Morawiecki spricht sich auf einer Pressekonferenz für höhere Gehälter für Lehrer aus. } \\
\text { Den Vorsitzenden der Gewerkschaft der Polnischen Lehrerschaft (Związek Nauczycielstwa Polskiego - ZNP), } \\
\text { Sławomir Broniarz, kritisiert er, politische Entscheidungen erpressen zu wollen. Vor dem Hintergrund eines groß- } \\
\text { angelegten Lehrerstreiks, über den zurzeit abgestimmt wird, hatte Broniarz vor zwei Tagen in einem Interview } \\
\text { gesagt, dass die Schüler möglicherweise ihre Abschlussprüfungen nicht termingerecht würden ablegen können. }\end{array}$ \\
\hline
\end{tabular}

Sie können die gesamte Chronik seit 2007 auch auf http://www.laender-analysen.de/polen/ unter dem Link "Chronik« lesen. 


\section{ÜBER DIE POLEN-ANALYSEN}

Die Polen-Analysen erscheinen zweimal monatlich als E-Mail-Dienst. Sie werden gemeinsam vom Deutschen PolenInstitut Darmstadt, der Forschungsstelle Osteuropa an der Universität Bremen und der Deutschen Gesellschaft für Osteuropakunde herausgegeben.

Ein Archiv der Polen-Analysen finden Sie im Internet unter www.laender-analysen.de/polen Kostenloses Abonnement unter http://www.deutsches-polen-institut.de/Newsletter/subscribe.php

Diese Analysen finden Sie online als Lizenzausgabe auf bpb.de

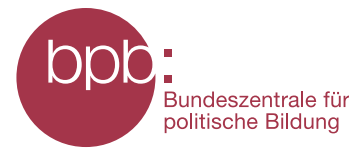

Deutsches Polen-Institut Darmstadt (www.deutsches-polen-institut.de)

Das seit 1980 tätige Deutsche Polen-Institut Darmstadt (DPI) ist ein Forschungs-, Informations- und Veranstaltungszentrum für polnische Kultur, Geschichte, Politik, Gesellschaft und die deutsch-polnischen Beziehungen, die sich im Kontext der europäischen Integration entwickeln. Institutionelle Träger des DPI sind das Land Hessen, die Kultusminister der Länder, das Auswärtige Amt und die Wissenschaftsstadt Darmstadt. Einen wesentlichen Beitrag zur Verwirklichung der Institutsziele leisten private Stiftungen. Ziel der Vermittlertätigkeit des DPI ist es, »die zu interessieren, auf die es politisch, wirtschaftlich, gesellschaftlich und kulturell im deutsch-polnischen Verhältnis ankommt (Leitlinien 1997). Es geht um die Entscheider und Multiplikatoren in Politik, Kultur, Bildung, Verwaltung, Medien und Wirtschaft. Das DPI versteht sich in Kooperation mit den Orten wissenschaftlicher Polen-Kompetenz an deutschen Hochschulen und Forschungsinstituten als verbindendes und vernetzendes Zentrum. Mit der 70.000 Bände zählenden multidisziplinären Fachbibliothek für Polen, die eine einzigartige Sammlung polnischer Belletristik in der Originalsprache und in deutscher Übersetzung umfasst, ist das DPI ein geschätzter Ort der Recherche und des wissenschaftlichen Arbeitens.

Forschungsstelle Osteuropa an der Universität Bremen (www.forschungsstelle.uni-bremen.de)

1982 gegründet, widmet sich die Forschungsstelle Osteuropa an der Universität Bremen der interdisziplinären Analyse der Länder Ost- und Ostmitteleuropas in Zeitgeschichte und Gegenwart. Der Forschungsschwerpunkt liegt dabei auf der Rolle von »Dissens und Konsens«, von Opposition und Zivilgesellschaft in ihrem historischen, politischen, gesellschaftlichen und kulturellen Kontext. Die Forschungsstelle besitzt in ihrem Archiv eine einzigartige Sammlung alternativer Kulturgüter und unabhängiger Texte aus den ehemaligen sozialistischen Ländern. Darunter befindet sich auch eine umfangreiche Sammlung des "Zweiten Umlaufs«, die das Schrifttum und Dokumente unabhängiger Initiativen und gesellschaftlicher Gruppen in Polen aus der Zeit von 1976 bis zum Umbruch umfasst. Hinzu kommt eine umfangreiche Bibliothek mit wissenschaftlicher Literatur. Mit Archiv, Bibliothek und zwei wissenschaftlichen Abteilungen ist die Forschungsstelle auch eine Anlaufstelle sowohl für Gastwissenschaftler als auch für die interessierte Öffentlichkeit.

Eine der Hauptaufgaben der Forschungsstelle ist die Information der interessierten Öffentlichkeit. Dazu gehören unter anderem regelmäßige E-Mail-Informationsdienste für Politik, Wirtschaft, Zivilgesellschaft und Medien.

Herausgeber:

Deutsches Polen-Institut, Deutsche Gesellschaft für Osteuropakunde e.V., Forschungsstelle Osteuropa an der Universität Bremen, Leibniz-Institut für Agrarentwicklung in Transformationsökonomien, Leibniz-Institut für Ost- und Südosteuropaforschung, Zentrum für Osteuropa- und internationale Studien (Z0iS) gGmbH

Redaktion:

Prof. Dr. Dieter Bingen (verantwortlich) (Darmstadt) und Silke Plate M.A. (Bremen)

Satz: Matthias Neumann

Wissenschaftlicher Beirat:

Prof. Dr. Stefan Garsztecki, Technische Universität Chemnitz

Prof. Dr. Klaus Ziemer, Kardinal-Stefan-Wyszyński-Universität Warschau

Die Meinungen, die in den Polen-Analysen geäußert werden, geben ausschließlich die Auffassung der Autoren wieder. Abdruck und sonstige publizistische Nutzung sind nach Rücksprache mit der Redaktion gestattet. Polen-Analysen-Layout: Cengiz Kibaroglu, Matthias Neumann

Alle Ausgaben der Polen-Analysen sind mit Themen- und Autorenindex archiviert unter www.laender-analysen.de

ISSN 1863-9712 @ 2019 by Deutsches Polen-Institut, Deutsche Gesellschaft für Osteuropakunde e.V., Forschungsstelle Osteuropa an der Universität Bremen, Leibniz-Institut für Agrarentwicklung in Transformationsökonomien, Leibniz-Institut für Ost- und Südosteuropaforschung, Zentrum für Osteuropa- und internationale Studien (ZOiS) gGmbH

Kontakt: Dr. Andrzej Kaluza, Presse- und Öffentlichkeitsarbeit, Deutsches Polen-Institut, Residenzschloss, Marktplatz 15

64283 Darmstadt, Tel.: +49/6151/4202-20, Fax: +49/6151/4202-10, E-Mail: info@dpi-da.de, Internet: www.laender-analysen.de/polen 


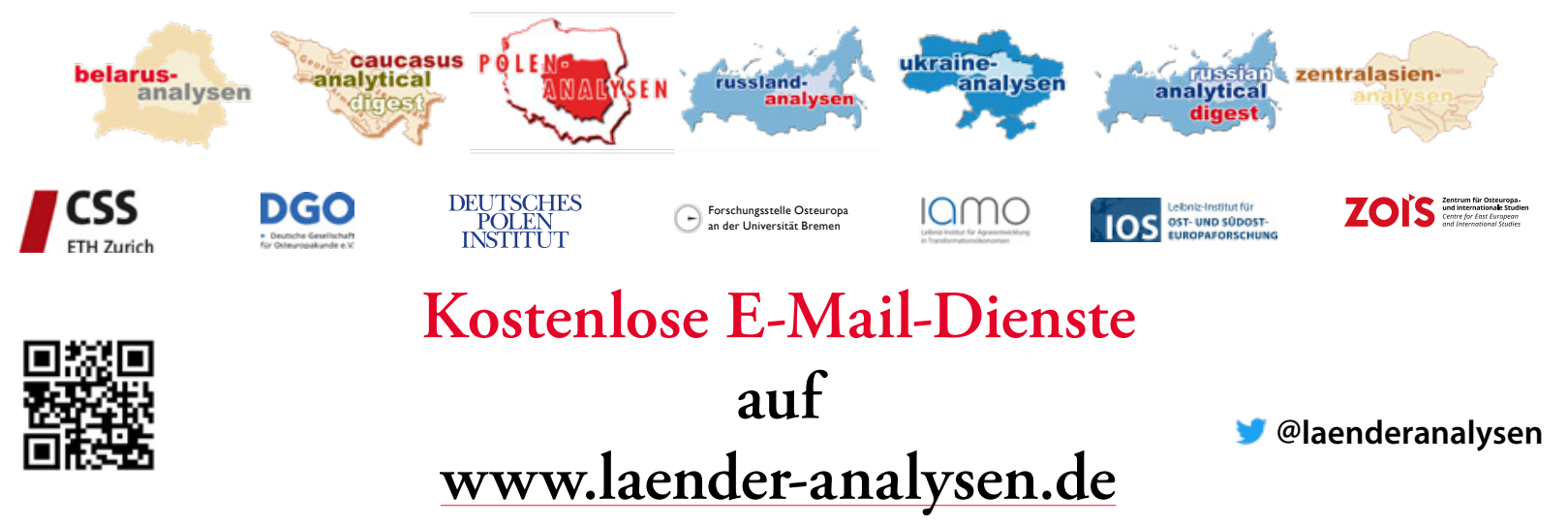

Die Länder-Analysen bieten regelmäßig im kostenlosen Abonnement kompetente Einschätzungen aktueller politischer, wirtschaftlicher, sozialer und kultureller Entwicklungen in Ostmitteleuropa und der GUS. Alle Länder-Analysen verstehen sich als Teil eines gemeinsamen Projektes, das der wissenschaftlich fundierten, allgemeinverständlich formulierten Analyse der Entwicklungen im östlichen Europa, der Offenheit für verschiedene inhaltliche Positionen und der kostenlosen und nicht-kommerziellen Information einer breit verstandenen interessierten Öffentlichkeit verpflichtet ist. Autor/innen sind internationale Fachwissenschaftler/innen und Expert/innen. Die Redaktionen der Länder-Analysen bestehen aus Wissenschaftler/innen mit langjähriger Forschungserfahrung.

Die deutschsprachigen Länder-Analysen werden gemeinsam von der Forschungsstelle Osteuropa an der Universität Bremen, dem Zentrum für Osteuropa- und internationale Studien, der Deutschen Gesellschaft für Osteuropakunde, dem Deutschen Polen-Institut, dem Leibniz-Institut für Agrarentwicklung in Transformationsökonomien und dem Leibniz-Institut für Ost- und Südosteuropaforschung herausgegeben. Die englischsprachigen Länder-Analysen erscheinen in Kooperation der Forschungsstelle Osteuropa mit dem Center for Security Studies (CSS) der ETH Zürich.

Die Länder-Analysen bieten regelmäßig Kurzanalysen zu aktuellen Themen, ergänzt um Grafiken und Tabellen sowie Dokumentationen. Zusätzlich gibt es eine Chronik aktueller Ereignisse. Alle Länder-Analysen sind auch mit Archiv und Indizes online verfügbar unter www.laender-analysen.de.

\section{Belarus-Analysen}

Erscheinungsweise: zweimonatlich

Abonnement unter: http://www.laender-analysen.de/belarus/

\section{Caucasus Analytical Digest}

In englischer Sprache. Erscheinungsweise: zweimonatlich

Abonnement unter: http://www.css.ethz.ch/en/publications/cad.html

\section{Polen-Analysen}

Erscheinungsweise: zweimal monatlich

Abonnement unter: http://www.deutsches-polen-institut.de/newsletter/polen-analysen/ Auch als App für Android ${ }^{\mathrm{TM}}$ (ab Januar 2016) kostenlos auf Google Play ${ }^{\mathrm{TM}}$.

\section{Russland-Analysen}

Erscheinungsweise: zweimal monatlich

Abonnement unter: http://www.laender-analysen.de/russland/

Auch als App für Android (ab Januar 2016) kostenlos auf Google Play.

\section{Russian Analytical Digest}

In englischer Sprache. Erscheinungsweise: zweimal monatlich Abonnement unter: http://www.css.ethz.ch/en/publications/rad.html

\section{Ukraine-Analysen}

Erscheinungsweise: zweimal monatlich

Abonnement unter: http://www.laender-analysen.de/ukraine/

Auch als App für Android (ab Januar 2016) kostenlos auf Google Play.

\section{Zentralasien-Analysen}

Erscheinungsweise: zweimonatlich

Abonnement unter: http://www.laender-analysen.de/zentralasien/ Auch als App für Android (ab Januar 2016) kostenlos auf Google Play.

Google Play, das Google Play-Logo und Android sind Marken von Google Inc. TWITTER, TWEET, RETWEET und das Twitter Logo sind eingetragene Markenzeichen von Twitter, Inc. oder angeschlossenen Unternehmen. Der Android-Roboter wird auf der Grundlage von einem Werk wiedergegeben oder modifiziert, das von Google erstellt und geteilt und gemäß den Bedingungen der Creative Commons 3.0 Attribution-Lizenz verwendet wird. 